\title{
FAVORECER LA COLABORACIÓN POR MEDIO DE LAS TIC:EL NUEVO DESAFÍO DE LA FORMACIÓN PRÁCTICA DE LOS FUTUROS PROFESORES Y PROFESORAS
}

\author{
Thierา Karsenti*
}

El presente estudio contribuve a la comprensión de las ventajas del uso de Tecnologías de Información y. Comunicación (TIC) en la formación práctica de maestros de secundaria, en el contexto de un programa de formación de la Universidad de Quebec en Hull (Canadá). Mediante una metodología de análisis cualitativo, se analizan los mensajes de dos grupos de participantes inmersos en un entorno de aprendizaje colaborativo con integración de TIC (sitio Web multimedia-foro electrónico de discusión). El análisis del contenido de la muestra de mensajes, resultante de las interacciones de los participantes, destaca el intercambio de experiencias de aprendizaje en situaciones de la práctica escolar diaria, por ejemplo: compartir experiencias personales, retroalimentación, consejos pedagógicos, estímulos y solidaridad, principalmente, pero también el nulo cuestionamiento de sus posiciones. Las numerosas colaboraciones atestiguan que los intercambios y las experiencias compartidas, constituyen una comunidad de aprendizaje, en la que los maestros se sienten revalorizados y con mayor confianza para el desarrollo de su competencia.
This article contributes to the understanding of the adiantages of using communication and information technologies in practical training of secondary teachers in the context of a teacher training program of the University of Quebec in Hull, Canada. Utilizing a qualitative analysis methodology; the messages of two groups of teaching students are analyzed in a context of a collaborative learning, integrating communication and information technologies (electronic multimedia-forum Web-site). The content analysis of the messages sample resulting from teaching students interactions points out the learning experiences exchange on school daily situations, for example: sharing personal experiences, professional feedback pedagogic counseling, stimuli and solidarity; in addition, the scarce of their questioning personal positions. Numerous collaborations testify that the professional exchanges and the experiences shared conform a learning community, in which teachers feel stimulated and more confident for developing their professional competence.

* Es catedrático y enseña el curso Tecnologías de Información y Comunicación (TIC) en Educación en la Universidad de Montreal. También enseña cursos en el campo de tecnologías de información y comunicación y su integración en la formación docente. Su desempeño en innovación de tecnología educativa es reconocido a nivel regional y nacional. Fue ganador del primer lugar del concurso del Premio del Ministerio de Educación (1998-1999 y 1999-2000). Junto con un equipo de la Liniversidad de Quebec en Hull, recibió el Premio Homenaje 2001 del Gobiemo de Quebec. También, fue ganador del Premio de Excelencia en la Concepción Pedagógica, de la Asociación Canadiense de Educación a Distancia. así como el Premio PEDAGÓGICA-RESCOL de innovación pedagógica en la integración de TIC(2000). 
El presente estudio tiene por objetivo general comprender las ventajas potenciales de una integración más global de las TIC (tecnologías de la información y la comunicación) en la formación práctica de los futuros profesores. Específicamente, este estudio aspira a comprender mejor los tipos de interacción favorecidos, promovidos por un entorno de aprendizaje colaborativo sostenido por las TIC, entorno que toma la forma del despliegue de un sitio web multimedia con un foro electrónico de discusión ${ }^{1}$. Los resultados que en este artículo serán presentados, surgen de la colecta de datos hecha en paralelo a la implementación de una experiencia piloto de integración de las TIC, en el marco de la formación práctica de los futuros profesores de secundaria. Esperamos que una mejor comprensión de los tipos de interacción generados por un entorno de aprendizaje colaborativo, sustentado por las TIC. pueda contribuir al avance de los conocimientos en el plano de una integración más global de las nuevas tecnologías en la formación de maestros.

Debemos mencionar, que en Québec, hasta donde sabemos, sólo Nault $(2000)^{2}$ ha estudiado el fenómeno de integración de las TIC en la formación práctica de los futuros profesores. De cualquier manera, nuestra experiencia piloto se distingue de la experiencia de Nault (2000) en que, dado un contexto particular, las visitas de supervisión eran reemplazadas por un seguimiento sostenido por las TIC. El contexto y la tradición de la supervisión de practicantes en la Universidad de Québec en Hull, no nos permitiría tal cambio, al menos no en el corto plazo.

Nuestra presentación tratará, inicialmente, del contexto de la formación práctica en la Universidad de Québec en Hull, contexto que ha favorecido la integración de las TIC. Presentaremos de manera suscinta, el marco teórico que ha servido de base al análisis de los vínculos de colaboración favorecidos por esta experiencia piloto. Finalmente, encontraremos la presentación y análisis de los resultados, seguidos de una conclusión y de pistas futuras de investigación.

\section{El contexto de la experiencia}

\section{Las prácticas de intervención y la formación práctica de los futuros maestros}

La formación práctica, en los programas del ciclo básico para la formación de profesores de secundaria, ocupa un lugar importante en las universidades de Québec: unos ciento veinte días de práctica (como período de prueba) en las escuelas, repartidos en los cuatro años de formación, permite a los estudiantes desarrollar progresivamente sus competencias profesionales en sus respectivas

\footnotetext{
1 http://www.uqah.uquebec.ca/stages o http://stages.scedu.umontreal.ca

2 También en Nault \& Nault (2001).
} 
disciplinas. Para Nault \& Nault (2001), la práctica de prueba es la ocasión para el futuro maestro, de ejercer en el medio escolar. Cada vez más, como lo señala el Ministerio de Educación de Québec:

La concepción de un programa de formación docente centrado en el desarollo de competencias profesionales, debiera estar enraizada en los lugares de práctica. Las prácticas iniciales o de prueba en un medio escolar, constituyen ocasiones privilegiadas para ejercer las competencias en contexto real y evaluar su grado de progresión y adquisición. Es indispensable que los establecimientos de enseñanza continúen proveyendo a los candidatos al oficio docente, la posibilidad de vivir experiencias de terreno. Los futuros maestros pueden tener, entonces, la ocasión de demostrar que poseen las competencias requeridas para ejercer la profesión. (MEQ, 2001b, p.217)

De la etapa de observación en internado, pasando por la preparación didáctica y la toma a cargo de una situación de clase, la formación práctica compromete a los practicantes a frecuentar varias escuelas a lo largo de su programa. Para lograrlo, las universidades pueden, en general, contar con la colaboración de una red de escuelas asociadas que se extiende en un vasto territorio, lo que permite a los estudiantes el beneficiarse de experiencias prácticas en medios escolares diversos. Por ejemplo, de un trimestre al otro, varios practicantes son ubicados a distancias considerables de sus universidades de origen, esto es cierto en particular para las universidades situadas en regiones, tal como la Universidad de Québec en Hull.

Nault \& Nault (2001) destacan que los supervisores son a menudo llamados a ausentarse del medio universitario para ir a observar las clases de los practicantes de los cuales son responsables, aún si los practicantes están ubicados en diferentes escuelas, a menudo muy alejadas unas de otras. Estas condiciones de grandes desplazamientos en un número importante de visitas a efectuar, también presentes en la universidad de Québec en Hull, hacían más difícil el mantener una colaboración activa con los profesores asociados y los practicantes en cada visita de observación y de evaluación. Es ese contexto el que nos ha animado, por una parte, a querer integrar las TIC en el marco de la formación práctica de los futuros profesores. Es, luego, a partir de esta experiencia de integración de las TIC, que hemos querido comprender cómo se desarrollan los tipos de interacción que han sido favorecidos por un entorno de aprendizaje colaborativo, sostenido por las nuevas tecnologías. En este caso miraremos el despliegue de un sitio web multimedia con un foro electrónico de discusión (objetivo del presente estudio). 


\section{Marco teórico}

En función de los principales modelos encontrados en la literatura, y que aspiran a comprender mejor los tipos de interacción generados en el momento del aprendizaje colaborativo, entre estos los modelos de Berge (1995). de Moore (1993), de Tuovunen (2000), de Johnson y Johnson (1998) y de CecezKecmanovic y Webb (2000), nos proponemos elaborar un modelo de síntesis que nos permita comprender mejor el tipo de interacciones presentes en un ambiente de aprendizaje colaborativo sustentado por las TIC.

De los modelos de Berge, Moore y Tuovinen, retenemos dos categorías de análisis:

- las interacciones aprendiz-formador;

- las interacciones aprendiz-aprendiz.

Del modelo de Johnson y Johnson (1998), retenemos los cuatro elementos siguientes:

1. Dar y recibir ayuda.

2. Intercambiar recursos o información.

3. Dar y recibir «feedback».

4. Cuestionar los posicionamientos.

Del modelo de Cecez-Kecmanovic y Webb (2000), retenemos los dos elementos siguientes:

1. Acciones liguísticas que han tratado de la materia aprendida o de un tema de discusión.

2. Acciones linguísticas vinculadas a experiencias personales, deseos, sentimientos.

La yuxtaposición de estos tres modelos nos induce a proponer un nuevo modelo (cuadro 1) en el cual una misma interacción es analizada bajo dos grandes categorías, la del tipo de interacción (aprendiz-aprendiz, aprendiz-formador) y la de la manifestación que ella toma (necesidad de ayuda, intercambio de recursos o de información, feedback, cuestionamiento de las posturas asumidas, compartir de experiencias personales, entre otras.) Nos parece que este modelo integra los principales elementos retenidos de todos los anteriores, y de manera creciente, la categoría « otras » permitiría a un potencial contenido emerger de los análisis. por ejemplo por el método de la teoría anclada (Savoie-Zajc, 2000). 


\section{Cuadro 1}

Modelo de análisis de las interacciones en un contexto de aprendizaje colaborativo sustentado por las TIC

\begin{tabular}{|l|l|l|l|l|l|}
\hline & $\begin{array}{c}\text { Manifestación } \\
\text { de la necesidad } \\
\text { de ayuda }\end{array}$ & $\begin{array}{c}\text { Intercambio } \\
\text { de recursos o } \\
\text { de información }\end{array}$ & Feedback & $\begin{array}{c}\text { Cuestionamiento } \\
\text { de los } \\
\text { posicionamientos }\end{array}$ & Otras \\
\hline $\begin{array}{l}\text { Interacciones } \\
\text { aprendiz- } \\
\text { aprendiz }\end{array}$ & & & & & \\
\hline $\begin{array}{l}\text { Interacciones } \\
\text { aprendiz- } \\
\text { formador }\end{array}$ & & & & & \\
\hline
\end{tabular}

\section{Metodología}

\section{Tipos de investigación}

Puesto que esta investigación aspira esencialmente a la comprensión en profundidad del fenómeno de la interacción, favorecido por el entorno de aprendizaje colaborativo que hemos generado, el tratamiento y análisis de datos es, ante todo, de tipo cualitativo. El análisis de datos se inspira de los modelos propuestos por L’Écuyer (1990), Sedlack \& Stanley (1992) y Huberman \& Miles (1991, 1994). Hemos privilegiado un tratamiento del tipo « análisis de contenido », basado en el modelo teórico de análisis de las interacciones, modelo que hemos desarrollado en función de una revisión crítica de la literatura sobre los análisis de las interacciones (cuadro 2). Según Sedlack \& Stanley (1992) y L'Écuyer (1990), el análisis de contenido es un «método de clasificación o de codificación de diversos elementos del material analizado, que permite al usuario conocer mejor las características y la significación » [del contenido] (L’Écuyer, 1990 ; p.9). Nos parece importante destacar que el análisis cualitativo de los textos de los mensajes electrónicos, se manifiesta como una vía promisoria, fuente de información en educación, particularmente con los medios didácticos y los entornos de aprendizaje propuestos a los estudiantes en los cursos en linea (Winiecki, 1999).

Los resultados de nuestro estudio se fundamentan en el análisis de mensajes electrónicos $(n=392)$ puestos en un foro de discusión de las mismas características, accesible por el sitio Web que habíamos creado para las prácticas docentes iniciales. 


\section{Muestreo}

Si bien la experiencia piloto se desarrolla desde hace sólo cuatro años y que unos 1400 practicantes ya han participado, nuestro análisis se basará únicamente en las interacciones registradas al interior de dos grupes de practicantes en su segundo año de formación $(n=82)$ los que jamás habían experimentado un tal entorno de aprendizaje. Como ya lo señalamos, el corpus analizado, contiene 392 mensajes electrónicos. El taller de práctica II en la Universidad de Québec en Hull, tiene igualmente la ventaja de estar estructurado en cuatro semanas, lo que facilitó el análisis de las interacciones durante la formación práctica en las escuelas, de los futuros profesores. Quisimos ver igualmente si, a pesar del corto lapso de tiempo, las TIC podían tener un impacto en la colaboración y en los tipos de interacción.

\section{Presentación y análisis de resultados}

El análisis interactivo de los resultados, efectuado a partir de un primer muestreo de 50 mensajes (de un total de 392) puestos en el foro de discusión, nos ha llevado a incluir tres nuevas categorías en el cuadro de análisis de contenido que habíamos elaborado a partir de la teoría. Estas cuatro nuevas categorías han «emergido» de manera significativa, a partir de los análisis interactivos de contenido de los 50 primeros mensajes. Ellas son :

- Consejos pedagógicos.

- Estímulo y solidaridad.

- Pedidos de feedback.

Al comienzo las habíamos agrupado sólo bajo la categoría «feedback», pero el análisis de varios mensajes nos condujo a distinguirlas. Estas nuevas categorías parecían pertinentes en el contexto de nuestra experiencia piloto, razón por la cual decidimos agregarlas al cuadro de análisis, ahora modificado (cuadro 2). Estas categorías no han reemplazado a la categoría « otras », dado que ciertos mensajes no eran susceptibles de ser reagrupados.

Así mismo, decidimos no tomar en consideración la distinción « aprendizaprendiz » y aprendiz-formador ». La estructura del foro estaba concebida -y sobre todo utilizada- de tal forma que los estudiantes practicantes «pegaban » sus mensajes, asumiendo que estos estaban a menudo destinados a sus colegas, pero conscientes, sin embargo, que esos mensajes eran leídos por el supervisor 
de práctica y, en ocasiones, por los profesores asociados (en el rol de tutores). Además, los participantes habían sido incitados a colocar sus intervenciones en el foro electrónico, de modo tal que no solamente quien ejerciera la supervisión pudiese responder, sino también los otros aprendices.

El cuadro 2 presenta una definición de cada categoría, con un ejemplo de mensaje puesto en el foro electrónico de discusión y que ilustra claramente la respectiva categoría de análisis. Los ejemplos son tomados del conjunto de mensajes colocados en dicho foro $(n=392)$.

\section{Cuadro $\mathbf{N}^{\circ} 2$}

\section{Definición de las categorías de análisis de contenido de los mensajes puestos en el Foro de discusión y presentación de un ejemplo}

\begin{tabular}{|l|l|l|}
\hline $\begin{array}{l}\text { Manifestación de la } \\
\text { necesidad de ayuda }\end{array}$ & $\begin{array}{l}\text { Definición sumaria } \\
\text { Un practicante pide } \\
\text { formalmente la ayuda de sus } \\
\text { pares o del supervisor a fin } \\
\text { de resolver una situación } \\
\text { difícil. }\end{array}$ & $\begin{array}{l}\text { Chantal (nombre ficticio) } \\
\text { escribe: «...Hay alguien } \\
\text { que tenga alguna idea } \\
\text { genial? Es muy utópico } \\
\text { creer que un grupo de } \\
\text { alumnos de quinto año de } \\
\text { secundaria pueda trabajar } \\
\text { en equipo manteniendo un } \\
\text { nivel de ruido tolerable? } \\
\text { Les he pedido su opinión y } \\
\text { sugerencias y parece que no } \\
\text { hay esperanza Por favor, } \\
\text { díganme que no es verdad.» }\end{array}$ \\
\hline $\begin{array}{l}\text { Intercambio de recursos o } \\
\text { de información }\end{array}$ & $\begin{array}{l}\text { Un practicante que informa a } \\
\text { sus pares sobre un evento, } \\
\text { una actividad o un tipo de } \\
\text { intervención usada por su } \\
\text { profesor tutor (asociado) o } \\
\text { por su escuela. Se trata de } \\
\text { una información en el } \\
\text { sentido más propio del } \\
\text { término. El practicante } \\
\text { informa (a sus pares). }\end{array}$ & $\begin{array}{l}\text { Julie (nombre ficticio) } \\
\text { escribe: «...Todos los años } \\
\text { [la escuela de práctica] } \\
\text { ofrece en el mes de mayo } \\
\text { una exposición de todas las } \\
\text { obras de alumnos en artes } \\
\text { plásticas. Esta exposición } \\
\text { está abierta a todo público } \\
\text { y permite reunir fondos } \\
\text { para la escuela. Prometo ir } \\
\text { a echar un vistazo y } \\
\text { ustedes? }\end{array}$ \\
\hline
\end{tabular}

3 Todos los nombres propios en los mensajes son ficticios. Han sido modificados para proteger el anonimato de los practicantes. 


\begin{tabular}{|c|c|c|}
\hline & Definición sumaria & Presentación de un ejemplo \\
\hline Consejos pedagógicos & $\begin{array}{l}\text { Un practicante que otorga un } \\
\text { consejo de orden pedagógico } \\
\text { a sus pares, que pronuncia } \\
\text { una moraleja a retener luego } \\
\text { de una situación pedagógica } \\
\text { vivida u observada; que } \\
\text { propone un modo de actuar } \\
\text { en lo pedagógico a fin de } \\
\text { evitar ciertos problemas; que } \\
\text { propone una estrategia, una } \\
\text { forma de abordar una } \\
\text { situación o un tipo particular } \\
\text { de intervención pedagógica. } \\
\text { Puede tratarse igualmente de } \\
\text { un practicante que emite una } \\
\text { opinión sobre alguna materia. }\end{array}$ & $\begin{array}{l}\text { Sophie (nombre ficticio) } \\
\text { escribe: «...Creo que es } \\
\text { primordial tener siempre } \\
\text { bajo la manga una solución } \\
\text { alternativa. Cada vez más } \\
\text { me percato que la } \\
\text { capacidad de adaptación es } \\
\text { una cualidad esencial en un } \\
\text { buen profesor.» } \\
\text { Julien (nombre ficticio) } \\
\text { escribe: «Nada mejor que } \\
\text { un evento escolar para } \\
\text { acercarse a los alumnos a } \\
\text { los que se enseña...» }\end{array}$ \\
\hline Estímulos y « solidaridad » & $\begin{array}{l}\text { Un practicante que estimula, } \\
\text { que motiva a sus pares, que } \\
\text { invita en su mensaje a «no } \\
\text { relajarse», a perseverar, etc., } \\
\text { sin que por eso su mensaje } \\
\text { sea una respuesta a otro } \\
\text { compañero. Se trata de un } \\
\text { mensaje general de estímulo } \\
\text { o de solidaridad. (Tipo de } \\
\text { interación a menudo } \\
\text { encontrada al inicio o fin } \\
\text { del mensaje.) }\end{array}$ & $\begin{array}{l}\text { Isabelle (nombre ficticio) } \\
\text { escribe: «Espero que todo } \\
\text { vaya bien para ustedes, no } \\
\text { desfallezcan, tenemos una } \\
\text { buena parte de la práctica } \\
\text { realizada...» }\end{array}$ \\
\hline Pedidos de feedback & $\begin{array}{l}\text { Un practicante que pide a sus } \\
\text { pares responder a su mensaje } \\
\text { a fin de obtener opiniones } \\
\text { sobre una situación, o saber } \\
\text { si la han vivido o si } \\
\text { comparten su visión sobre la } \\
\text { misma. No se trata } \\
\text { exactamente de un pedido de } \\
\text { ayuda, sino de un deseo de } \\
\text { «intercambio» de ideas con } \\
\text { sus pares }\end{array}$ & $\begin{array}{l}\text { Paul (nombre ficticio) } \\
\text { escribe: «...Encuentro que } \\
\text { un local de reorientación } \\
\text { disciplinaria es fuerte como } \\
\text { práctica de solución } \\
\text { inmediata en caso de crisis. } \\
\text { No se entonces qué pensar } \\
\text { de este sistema de gestión. } \\
\text { Ustedes, ¿qué piensan?» }\end{array}$ \\
\hline
\end{tabular}




\begin{tabular}{|c|c|c|}
\hline & Definición sumaria & Presentación de un ejemplo \\
\hline Consejos pedagógicos & $\begin{array}{l}\text { Un practicante que otorga un } \\
\text { consejo de orden pedagógico } \\
\text { a sus pares, que pronuncia } \\
\text { una moraleja a retener luego } \\
\text { de una situación pedagógica } \\
\text { vivida u observada; que } \\
\text { propone un modo de actuar } \\
\text { en lo pedagógico a fin de } \\
\text { evitar ciertos problemas; que } \\
\text { propone una estrategia, una } \\
\text { forma de abordar una } \\
\text { situación o un tipo particular } \\
\text { de intervención pedagógica. } \\
\text { Puede tratarse igualmente de } \\
\text { un practicante que emite una } \\
\text { opinión sobre alguna materia. }\end{array}$ & $\begin{array}{l}\text { Sophie (nombre ficticio) } \\
\text { escribe: «...Creo que es } \\
\text { primordial tener siempre } \\
\text { bajo la manga una solución } \\
\text { alternativa. Cada vez más } \\
\text { me percato que la } \\
\text { capacidad de adaptación es } \\
\text { una cualidad esencial en un } \\
\text { buen profesor.» } \\
\text { Julien (nombre ficticio) } \\
\text { escribe: «Nada mejor que } \\
\text { un evento escolar para } \\
\text { acercarse a los alumnos a } \\
\text { los que se enseña...» }\end{array}$ \\
\hline Estímulos y « solidaridad » & $\begin{array}{l}\text { Un practicante que estimula, } \\
\text { que motiva a sus pares, que } \\
\text { invita en su mensaje a «no } \\
\text { relajarse», a perseverar, etc., } \\
\text { sin que por eso su mensaje } \\
\text { sea una respuesta a otro } \\
\text { compañero. Se trata de un } \\
\text { mensaje general de estímulo } \\
\text { o de solidaridad. (Tipo de } \\
\text { interación a menudo } \\
\text { encontrada al inicio o fin } \\
\text { del mensaje.) }\end{array}$ & $\begin{array}{l}\text { Isabelle (nombre ficticio) } \\
\text { escribe: «Espero que todo } \\
\text { vaya bien para ustedes, no } \\
\text { desfallezcan, tenemos una } \\
\text { buena parte de la práctica } \\
\text { realizada...» }\end{array}$ \\
\hline Pedidos de feedback & $\begin{array}{l}\text { Un practicante que pide a sus } \\
\text { pares responder a su mensaje } \\
\text { a fin de obtener opiniones } \\
\text { sobre una situación, o saber } \\
\text { si la han vivido o si } \\
\text { comparten su visión sobre la } \\
\text { misma. No se trata } \\
\text { exactamente de un pedido de } \\
\text { ayuda, sino de un deseo de } \\
\text { «intercambio» de ideas con } \\
\text { sus pares }\end{array}$ & $\begin{array}{l}\text { Paul (nombre ficticio) } \\
\text { escribe: «... Encuentro que } \\
\text { un local de reorientación } \\
\text { disciplinaria es fuerte como } \\
\text { práctica de solución } \\
\text { inmediata en caso de crisis. } \\
\text { No se entonces qué pensar } \\
\text { de este sistema de gestión. } \\
\text { Ustedes, ¿qué piensan?» }\end{array}$ \\
\hline
\end{tabular}




\begin{tabular}{|l|l|l|}
\hline & \multicolumn{1}{|c|}{ Definición sumaria } & Presentación de un ejemplo $^{1}$ \\
\hline Otras & $\begin{array}{l}\text { Todo otro tipo de mensaje no } \\
\text { coincidente con las } \\
\text { categorías precedentes. }\end{array}$ & $\begin{array}{l}\text { Robert (nombre ficticio) } \\
\text { escribe: «Lindo día hoy, } \\
\text { ¿no creen?». } \\
\text { Julie (nombre ficticio) } \\
\text { escribe: «iBuenos días a } \\
\text { todos!» }\end{array}$ \\
\hline
\end{tabular}

En el cuadro $\mathrm{N}^{\circ} 3$, el nuevo esquema de análisis se presenta con la proporción de mensajes puestos en el foro electrónico de discusión, según cada categoría de pertenencia. El número de participantes en el foro es de 82 practicantes, todos los cuales, salvo uno, enviaron algún mensaje caracterizado por compartir en él experiencias personales. Este tipo de interacción se encuentra en el $72,7 \%$ de los mensajes puestos en el boletín electrónico ${ }^{4}$. Luego, encontramos igualados los porcentajes para los mensajes de feedback y de consejos pedagógicos, cuyas categorías fueron halladas en el $36,4 \%$ del total de los mensajes puestos respectivamente en el foro de discusión. Se observa luego los mensajes de estímulo, presentes en el $17,4 \%$ de las interacciones, seguidos de los mensajes referidos al intercambio de recursos o de información $(9,8 \%)$, al pedido de feedback $(9,1 \%)$ y finalmente, de los mensajes que manifiestan una necesidad, un pedido de ayuda $(5,3 \%)$. Asombra el constatar la ausencia de los cuestionamientos de posicionamiento, a diferencia de lo emergido en una propuesta teórica. Tal disposición al cuestionamiento había sido estimulado en los practicantes, sin embargo es posible que el contexto de formación práctica no se preste bien a esta forma de interacción. Ningún practicante parece cuestionar la posición adoptada por un colega frente a un evento o un problema dado. Todos parecen de acuerdo dentro del marco de pequeños matices de las posiciones originales. Los practicantes parecen comprenderse, vivir situaciones semejantes, estar de acuerdo con los propósitos de los otros.

4 Los mensajes puestos en el foro electrónico de discusión, podían, al mismotiempo, ser clasificados en varias categorías. Algunos mensajes eran particularmente largos y contenían, por ejemplo, mensajes de estímulo, consejos pedagógicos, la manifestación de necesidad de ayuda, etc. Todo de forma simultánea. 


\section{Cuadro 3 \\ Distribución porcentual de los mensajes puestos en el foro electrónico de discusión}

\begin{tabular}{|l|c|}
\hline Tipo de interacción & Porcentaje en relación al total de mensajes \\
\hline Compartir experiencias personales & $\mathbf{7 2 , 7 \%}$ \\
Feedback & $36,4 \%$ \\
Consejos pedagógicos & $36,4 \%$ \\
Estímulos y « solidaridad » & $\mathbf{1 7 , 4 \%}$ \\
Intercambio de recursos o de información & $\mathbf{9 , 8 \%}$ \\
Pedidos de feedback & $\mathbf{6 , 1 \%}$ \\
Manifestación de la necesidad de ayuda & $\mathbf{5 , 3 \%}$ \\
Otras & $\mathbf{5 , 3 \%}$ \\
Cuestionamiento de posicionamientos & $\mathbf{0 \%}$ \\
\hline
\end{tabular}

Resulta interesante el constatar que un cierto porcentaje (17.3\%) de los mensajes categorizados como un «compartir de experiencias personales », resultan estar integrados a los feedback que se otorgan a los colegas. En relación a los feedback, hay un buen número de practicantes que los otorgaron en más de una ocasión $(92,0 \%)$. Algunos, no más de dos veces, pero son varios los que escribieron más de cinco mensajes de este tipo $(64,1 \%)$, en tanto que otros enviaron hasta 9 feedbacks $(12,2 \%)$.

Un análisis detallado de los mensajes nos permite constatar que, a pesar de la distancia geográfica que separa a los practicantes, y como muchos lo han destacado al finalizar el período de práctica y a propósito de un seminario « no virtual » que los congregó presencialmente, una herramienta de este tipo les ha permitido acrecentar la solidaridad de grupo. La expresión de dicha solidaridad se refleja en casi la quinta parte de los mensajes. Es así como el 17,4\% de los mensajes puestos en el foro electrónico de discusión, contiene algún tipo de estímulo que refuerza la solidaridad. Encontramos, por ejemplo, mensajes del tipo:

«iFelicitaciones a todo el mundo; llevamos más de una semana!» «¡Buen término de práctica!»

«Vamos, ánimo todo el mundo [...]»

Como ya lo habíamos destacado, el compartir experiencias personales es sin duda el tipo de mensaje más representativo de los hallados en el sitio (72,7\%). Esto tal vez no resulta tan asombroso como parece, si consideramos que el objetivo del foro electrónico de discusión, puesto en el sitio Web de los practicantes, era, precisamente, permitir a los estudiantes compartir las 
experiencias vividas en sus prácticas. En el extracto de mensaje que presentamos a continuación, un estudiante comparte su experiencia de observador y de maestro aprendiz, frente a un alumno a quien se le ha prescrito (y suministrado) Ritalín. Las observaciones hechas por Nicolás (nombre ficticio) son interesantes y la situación vivida lo mueve a tomar una posición y a dar su opinión sobre el asunto.

\section{Título del mensaje: Son tan tranquilos...}

Mensaje puesto por: Nicolás (nombre ficticio), enviado el 4/02/2001

Buenos días queridos colegas. [...], daré mis impresiones en relación al uso del Ritalín en los alumnos. Comencemos, lógicamente, por el inicio... Desde el primer momento en el que observaba el desarrollo de las clases, noté la presencia de un alumno muy inquieto. Este bellaco alteraba el buen funcionamiento del grupo y requería de una atención permanente de parte del profesor. Esto contribuía a alterar la clase influenciando a los otros estudiantes al relajo y la ociosidad. Es por eso que me preparé a mantener con él la disciplina, en cuanto me hiciera cargo de la clase. Para mi sorpresa, él estaba muy tranquilo y haciendo los trabajos pedidos. Yo no osé hacer mío todo el mérito de tal cambio de actitud, discutiéndolo con mi profesor tutor (asociado). El me informó que este alumno comenzaba un tratamiento con Ritalín a fin de tranquilizarlo. Yo reflexioné sobre el tema y me pregunté sobre el derecho de otros de imponer el uso de drogas a un joven estudiante. Seguro que esto me facilita la tarea, pero a qué precio para el estudiante? El hecho de verlo amorfo y siempre fatigado me hizo tomar conciencia de la potencia del así llamado medicamento, el que, a mi parecer, encadenab a la verdadera persona. Terminaré por mencionar que el objetivo de este texto no es aportar una solución, sino hacer tomar conciencia de un problema creciente. [...]

Esta toma de posición contra el Ritalín generó muchas respuestas, en general favorables a la idea sostenida por Nicolás. Estas reacciones diversas, a veces matizadas como el extracto de la respuesta de Elodio (nombre ficticio), ponen en evidencia cómo un foro de discusión electrónica, favorece la colaboración entre los practicantes. Así, un ímpetu de solidaridad, de ayuda mutua, de sostén, de confidencialidad y de colaboración, se observa. 


\section{Título del mensaje : Re : Son tan tranquilos...}

Mensaje puesto por Elodio, enviado el 4/02/2001.

Hola Nicolás, estoy completamente de acuerdo contigo. El Ritalín puede ser eficaz para calmar a ciertos alumnos, pero no hay que impedir a los jóvenes vivir su juventud y expresarse. No se en qué año están, pero creo que en secundaria deben comenzar a comprender que tienen un rol que jugar en la preparación de su porvenir. Habría que procurar algo de modo que esos alumnos se pudieran destensar de un modo tal que no alteraran las clases, y creo que la educación física es excelente para ello (iclaro!) porque los alumnos se mueven y gastan energía. Entiendo que te hagas preguntas al respecto.

Otra de las ventajas de un Foro electrónico es que las respuestas son inmediatas. El mensaje de Nicolás (nombre ficticio) sobre el Ritalín, recibe muchas respuestas el mismo día. Es una señal de que los practicantes están conectados y que están atentos a lo que pasa en el foro de discusión. Es, igualmente, una indicación de que el foro electrónico de discusión favorece una colaboración y una comunicación crecientes, lo que permite a los practicantes compartir las experiencias vividas en sus prácticas o simplemente dar una opinión sobre algo que se discute.

Además de compartir sus experiencias personales y de responder a ciertos colegas, cerca de un tercio de los practicantes $(27,3 \%)$ llegó a dar un consejo de orden pedagógico a uno o más colegas. Estos consejos tenían que ver, sobre todo, con la gestión de la clase (ámbito en el que el tema de la disciplina en el aula es recurrente). Algunos «trucos », recetas o simplemente relatos de casos ejemplares, todo fundamentado en las experiencias vividas, observadas o « recibidas » (de otros), componen lo esencial de los mensajes encontrados. Por otra parte, en la lectura de esos consejos, constatamos no solamente que los practicantes han hecho una integración y una reflexión de sus vivencias, sino que además, observamos el anhelo de compartir esas experiencias con sus pares y de ofrecer los frutos de su aprendizaje a fin que, los que lo deseen, puedan aprovechar esos preciosos consejos. El foro electrónico de discusión ha permitido ese tipo de colaboración que consiste en un intercambi de experiencias prácticas. Los profesores en formación tienen, de este modo, acceso a los relatos de experiencias vividas por sus pares, aún si su práctica los sitúa en una escuela ubicada a varios kilómetros de distancia. Más aún, siguiendo el ejemplo del mensaje puesto por Gilles (nombre ficticio), varios practicantes comienzan a ser prolijos cuando se trata de dar consejos. 


\section{Título del mensaje: Los jóvenes...emotivos! \\ Mensaje puesto por Gilles, enviado el 4/04/2001.}

Durante una jornada, los adolescentes pasan por toda una gama de emociones. Alegría, cólera, pena, tristeza... Todos esos sentimientos van a reaparecer un día, ya sea en mi clase o en la casa. Durante la práctica $I$, dos chicas rompieron en llanto en mi presencia. ¿Qué hacer? [...] Hay que tener mucho cuidado, porque todo puede ser mal interpretado por los adolescentes. Entonces, yo simplemente debí hablarles [... ] Todo esto para mostrar que trabajamos con seres humanos. Hay que estar absolutamente a la escucha de nuestros alumnos... siempre poniendo atención, cuidado. Esto no resulta siempre fácil, pero los adolescentes lo agradecen. ¡Créanme!

Gilles.

La categoría Consejos pedagógicos, presente en el 36,54\% de los mensajes puestos en el foro electrónico de discusión y la categoría Estímulos y solidaridad, presente en el 17,4\% de las interacciones, son las que han emergido de modo más significativo de los análisis preliminares de datos. Estos resultados indican, posiblemente, la existencia de una fuerte solidaridad entre los practicantes y muestran al foro electrónico como uno de los lugares privilegiados para la expresión de dicha solidaridad. Este medio de comunicación electrónica presente en el sitio Web de las prácticas, permite a los estudiantes mantener el contacto, estrechar lazos. Un comentario espontáneo e interesante de parte de la profesora tutora (asociada) de un practicante nos resulta explícito al respecto :

«¡Qué solidaridad entre ustedes, estudiantes! ¡Es maravilloso a leery a vivir!...»

\section{Conclusion}

El sitio Web de las prácticas en secundaria con un foro electrónico de discusión: una experiencia piloto prometedora.

El análisis de los datos hasta hoy recopilados, nos lleva a creer que el sitio Web de las prácticas en secundaria, con un foro electrónico de discusión como dispositivo incorporado, contribuye a favorecer un fino marco estructural para los estudiantes en el momento de su formación práctica. Este entorno multimedia permite a los practicantes hacer un provechoso uso de una importante red de ayuda susceptible de maximizar su éxito escolar y de 
acrecentar su bienestar psicológico en situaciones, a veces difíciles de vivir. como las prácticas en las escuelas.

Gracias al este sitio Web. los estudiantes parecen también más involucrados en el proceso de aprendizaje. Su formación práctica nos ha parecido igualmente enriquecida por los medios de comunicación puestos a su disposición. los que han parecido favorecer la colaboración, la reflexión, el desarrollo de su pensamiento crítico. Esto posiblemente les ha permitido, con mayor facilidad. construir los conocimientos inherentes a su formación práctica y superar más fácilmente las dificultades encontradas.

Se trata entonces, de un recurso pedagógico y didáctico complementario que, según nosotros, no reemplaza de ningún modo la visita del supervisor en la sala de clases, pero que permite, no obstante, un marco estructural de la formación del futuro maestro, más rico, más intenso, más interactivo y, sobretodo, más colaborativo. Por otra parte, la experiencia piloto llevada a cabo con futuros profesores, relatada en este texto, ha mostrado que el sitio Web de las prácticas en secundaria con un foro de discusión, permite una comunicación y una colaboración acrecentadas, tanto entre el supervisor y el practicante, como entre el supervisor y el profesor tutor (asociado) o el director, o aún entre los propios practicantes. Numerosos son los que han apreciado tal modo de funcionamiento. que les permite intercambiar ideas, compartir dificultades y, en ciertos casos, superar los momentos difíciles (que son, al decir de la mayoría, más fáciles de vivir en tanto se sabe que otros experimentan también los mismos problemas).

La experiencia vivida hasta hoy, nos ha permitido constatar que los estudiantes también desarrollan el hábito de utilizar con más frecuencia las nuevas tecnologías y las funciones de información y de comunicación del sitio, tal como lo testimonia la frecuencia de las respuestas a los mensajes puestos en el foro. Nuestras observaciones sobre el número y el contenido de las intervenciones, son testimonios de una participación activa, alentadora, estimulante para los practicantes sea donde sea que estén. Las numerosas colaboraciones que testimonian claramente los intercambios y el compartir experiencias que aparecen en el foro, favorecen la solidaridad y la ayuda mutua. Sintiéndose apoyados por una comunidad aprendiz dinámica a la cual se asocian, los practicantes parecen sentirse más valorizados y más confiados en el desarrollo de sus competencias profesionales.

Las interacciones sociales sostenidas por las TIC, no tienen límites de tiempo o de espacio : ellas transforman y acrecientan substancialmente el espacio social de aprendizaje colaborativo. Las TIC son, entonces, para nosotros, uno de los desafíos que los responsables de la formación práctica, en particular en estas épocas de reformas, debieran aceptar, tanto en el plano de las innovaciones pedagógicas como en el plano de las pistas futuras de investigación. 
Referencias

Alavi, M. (1994). Computer-mediated collaborative learning: An empirical evaluation. MIS Quarterly, 18(2), 159-174.

Barker, B. (1986). Efforts to Improve the Preparation of Teachers for Rural Schools. ERIC Publications (ED265993).

Berge, Z. L. (1995). Facilitating computer Conferencing: Recommendations From the Field. Educational Technology. 35 (1), 22-30.

Berge, Z.L. (1995). Facilitating Computer Conferencing: Recommendations From the Field. Educational Technology. 35 (1) 22-30.

Bourbeau, L. (1997). La formation pratique à l'enseignement: l'organisation des stages en milieu scolaire : rapport d'enquête. Québec: FECS.

Bruner, J. (1990). Acts of Meaning. Boston, MA: Harvard University Press.

Cecez-Kecmanovic, D. \& Webb, C. (1999). Towards a Communicative Model of Collaborative Web-mediated Learning. ASCILITE 1999: Responding to Diversity, Brisbane (Australie), 5-8 décembre.

Cecez-Kecmanovic, D. and Webb, C. (2000) A critical inquiry into webmediated collaborative learning. In Aggarwal, A.K. (ed.) Web-based Learning: Opportunities and Challenges. Hershey, PA: the IDEA Group Publishing. p. 132-141.

Hiltz, S.R. (1995) The Virtual Classroom: Learning Without Limits via Computer Networks, New Jersey: Ablex Publishing Corp.

Huberman, A.M., \& Miles, M.B. (1991). Analyse des données qualitatives. Recueil de nouvelles méthodes. Bruxelles: De Boeck Université.

Huberman, A.M., \& Miles, M.B. (1994). Data Management and Analysis Methods. In N.K. Denzin \& Y.S. Lincoln (Eds.), Handbook of Qualitative Research. Thousand Oaks, CA: Sage Publication. 
Huynh, M.Q. (1999) A Critical Study of Computer-Supported Collaborative Learning. PhD, Binghamton, New York: SUNY.

Johanson, R. P.; Norland, D. L.; Olson, E.; Huth. L.; Bodensteiner, R. (1999). Internet and List-Serves To Support the Student Teaching Semester. ERIC publications (ED428060)

Johnson, D. W. \& Johnson R. T. (1996). Cooperation and the use of technology. In Handbook of Research for Educational Communication and Technology, ed. D. Jonassen. Macmillam, New York: Macmillan . p. 1017-1044.

Johnson, D. W. \& Johnson R. T. (1998). Cooperative Learning and Social Interdependence Theory. [document disponible en ligne]: http://www.clcrc.com/ pages/SIT.html. [30 juin, 2001].

Johnson, D. W., Maruyama, G., Johnson, R. T., Nelson, D., and Skon, L. (1981) 'Effect of cooperative, competitive, and individualistic goal structures on achievement: A meta analysis'. Psychological Bulletin, 89, 47-61.

Karsenti, T. (1999). Comment le recours aux TIC en pédagogie universitaire peut favoriser la motivation des étudiants: le cas d'un cours médiatisé sur le Web. Cahiers de la recherche en éducation, 6 (3) : 455-484.

Karsenti, T. et Larose, F. (2001). Les TIC... au cœur des pédagogies universitaires. Québec : Presses de l’Université du Québec.

Kecmanovic et Webb, 1999.

L'Écuyer, R. (1990). Méthodologie de l'analyse développementale de contenu. Méthode GPS et concept de soi. Sillery, Qc: Presses de l'Université du Québec.

Marttunen, M. (1998). Electronic mail as forum for argumentative interaction in higher education studies. Journal of Educational Computing Research. 18(4), p. 387-405.

Ministère de l'Éducation du Québec (2001a). La formation à l'enseignement : Les orientations, Les compétences professionnelles. Québec : Gouvernement du Québec. 
Ministère de l'Éducation du Québec (2001b) Programme de formation de l'école québécoise. Éducation préscolaire et enseignement primaire, Québec: Gouvernement du Québec.

Moore, M.G. (1993). Three types of interactions. In K. Harry \& M. Johns (Éds.). Distance education : New perspectives. Londres: Routledge. P. 19-24.

Mory, E. H. (1992). Use of information feedback in instruction implications for future research, Education Technology Research and Development, 40 (2), 40-43.

Nault, G. (2000). Exploration d'un dispositif de supervision des stagiaires via Internet. Mémoire de maîtrise. Montréal : Université du Québec à Montréal.

Nault, T. et Nault, G. (2001). Quand les stages attrapent les TIC. In Karsenti, T. et Larose, F., Les TIC... au cœur des pédagogies universitaires. Québec: Presses de l'Université du Québec.

Newman, D.R., Johnson, C., Webbs, B. \& Cochrane, C. (1997). Evaluating the quality of learning in computer supported co-operative learning. Journal of the American Society for Information Science. 48(6), 484-495.

Nipper, S. (1989). Third generation distance learning and computer conferencing. In R. Mason \& A. Kaye (Éds).). Mindweae: Communication, computers and distance education. Oxford (UK) : Pergamon. pp. 63-73.

O'Neill, A. (1996). Increasing reflective instructional decision-making by clinically supervising teachers using telecommunications. Proceedings of the Annual National Educational Computing Conference (Mineapolis, Minesota, 11-13 juin).

Piaget, J. (1962). Le langage et la pensée chez l'enfant. Paris: Presses universitaires de France.

Pugach, H \& Johnson, L.J. (1995) Collaborative practitioners and collaborative schools. Denver : Love Publishing Company.

Savoie-Zajc, L. (2000). La recherche qualitative. In Karsenti, T. et SavoieZajc, L., Introduction à la recherche en éducation. Sherbrooke: Éditions du CRP. 
Schlagal, B., Trathen, W. et Blanton, W. (1996). Structuring telecommunications to create instructional conversations about student teaching. Journal of Teacher Education, 47 (3), p. 175-183.

Searle, J.R. (1979). Expression and Meaning, Cambridge: Cambridge University Press.

Sedlack, R.G., \& Stanley, J. (1992). Social Research: Theory and Methods. Boston, MA: Allyn and Bacon.

Slavin, R.E. (1987). Cooperative Learning: Student Teams, Washington: National Educational Association.

Sringam, C. \& Geer, R. (2000). An Investigation of an Instrument for Analysis of Student-Led Electronic Discussions. ASCILITE 2000: Learning to choose, choosing to learn, Coffs Harbour (Australie), 9-14 décembre.

Stacey, E. (1998). Study of the enhancement of learning through group interaction by computer mediated communication. Thèse de doctorat. Monash University, Melbourne, Australie.

Tuovinen, J.E. (2000). Multimedia distance education interactions. Educational Media International, 37 (1), 16-24.

Venn, M. L.; Moore, R. L.; Gunter, P. L. (2001). Using Audio/Video Conferencing To Observe Field-Based Practices of Rural Teachers. Rural Educator; 22(2), p. 24-27.

Vygotsky, L.S. (1978) Mind in Society: The Development of Higher Psychological Processes, Cambridge, MA: Harvard University Press.

Winiecki, D. (1999). Studying and Analysing Asynchronous Conversations in Distance Education. Communication présentée lors du First International Interdisciplinary Conference Advances in Qualitative Methods (Edmonton, 1-2 mars).

Yaverbaum, G. J.; Ocker, R. J. (1998). Problem Solving in the Virtual Classroom: A Study of Student Perceptions Related to Collaborative Leaming Techniques. ERIC publications (ED427750).

Zeichner, K. (1992). Rethinking the Practicum in the Professional Development School Partnership. Journal of Teacher Education; 43(4), p. 296-307. 